\title{
EFFECT OF BISPHENOL-A ON THE MORPHOLOGY OF SMALL INTESTINE IN PREGNANT RATS
}

Jesmine Khan ${ }^{1 *}$, Siti Hamimah Sheikh Abdul Kadir ${ }^{1,2}$, Wan Nor I'zzah Wan Mohamad Zain ${ }^{1}$, Roziana Kamaludin $^{3}$, Zatilfarihah Rasdi ${ }^{2}$, Amirah Abdul Rahman $^{1}$

${ }^{1}$ Faculty of Medicine, Universiti Teknologi MARA, Cawangan Selangor, Kampus Sungai Buloh, Selangor, Malaysia

${ }^{2}$ Institute of Medical Molecular Biotechnology (IMMB), UiTM, Sungai Buloh, Selangor, Malaysia

${ }^{3}$ Advance Membrane Technologi (AMTEC), Universiti Teknologi Malaysia, Skudai, Johor, Malaysia

*Corresponding author: jesminek@yahoo.com

\section{Graphical abstract}

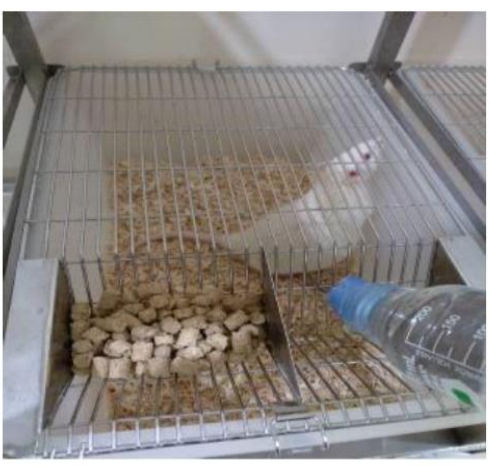

Pregnant rats receiving BPA in drinking water

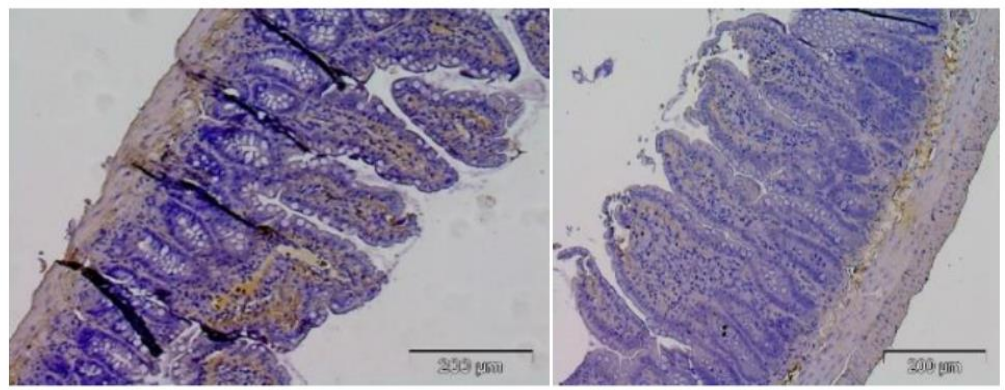

Expression of intestinal claudin 3 by Immunohistiochemical staining

\section{ABSTRACT}

Bisphenol A (BPA) is an environmental pollutant. Human beings are exposed to BPA through food and water. Due to its prolonged contact with the intestinal tract (IT), it might have harmful effects on the IT particularly in pregnant women and the fetuses. Objective of this study was to investigate the effect of BPA on the morphology and tight junction protein expression of the small intestine of pregnant rats. Twelve Pregnant Sprague Dawley rats were divided into group 1 (control, $n=6$ ) and group 2 (BPA treated, $n=6)$. Group 1 received tween 80 and group 2 received BPA $(0.2 \mathrm{mg} / \mathrm{ml})$ in drinking water. There 
were no significant differences in the villus height, crypt depth and the number of goblet cells in the jejunum and ileum between the two groups. Intestinal tight junction protein (ITJP) claudin 2 expression was similar in both groups. ITJPs claudin 3 and 4 were expressed less intensely in the ileum of group 2 as compared to group 1. Chronic low dose BPA throughout pregnancy in rats did not affect the morphology of villi, crypt, goblet cells and ITJP claudin 2 but reduced the expression of claudin 3 and 4 in the ileum, which might compromise intestinal barrier.

Keywords: Bisphenol A, villi, crypt, goblet cells, claudin 2, 3, 4

\section{Introduction}

Bisphenol A (BPA) is one of the most widely used industrial compounds worldwide.

It is mainly used in consumer products, such as epoxy resin lining food and beverage cans, plastic food containers, tablewares, baby feeding bottles and toys.

Chronic low dose exposure to BPA has several harmful effects in the body $(1,2,3)$

Gastrontestinal (GI) mucosa is in prolonged contact with BPA contaminated food and beverages before their absorption.

It is not known whether chronic exposure of the intestinal tract with BPA causes any GI mucosal injury.

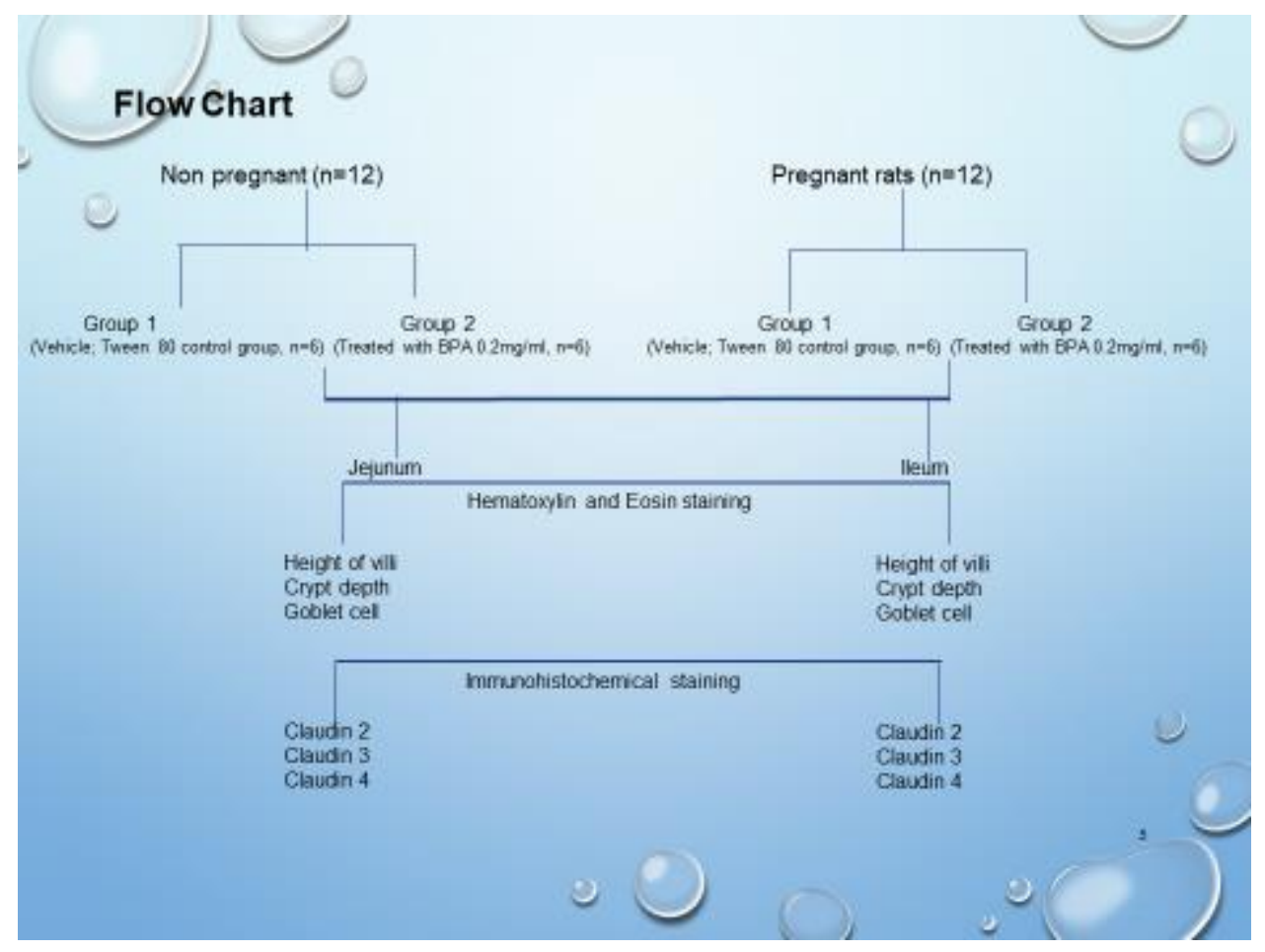




\section{RESULTS AND DISCUSSION}

There were no significant differences between the height and width of the villi, crypt depth and the number of goblet cells in the jejunum of group 1 and group 2 of pregnant and non pregnant rats.

A

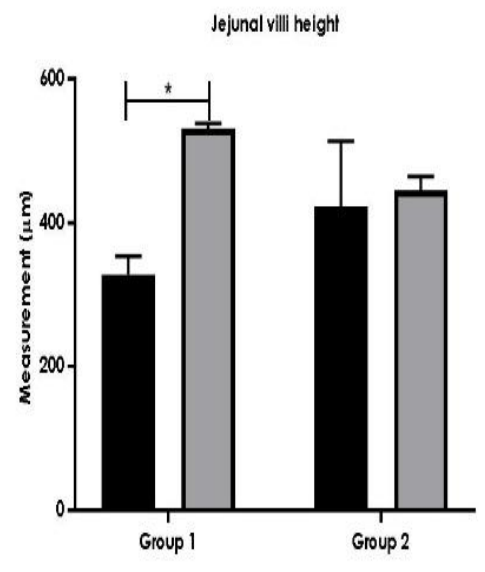

C

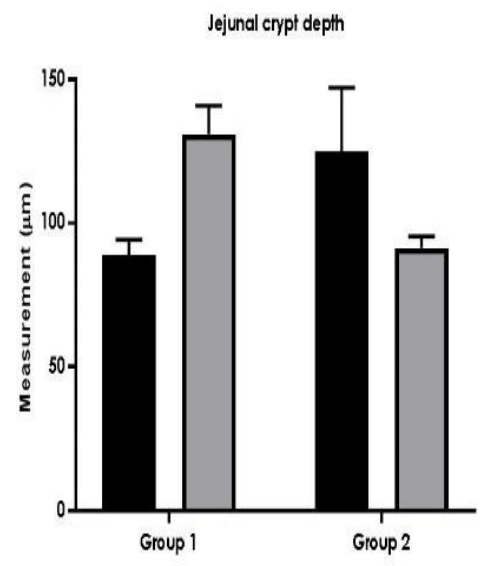

B

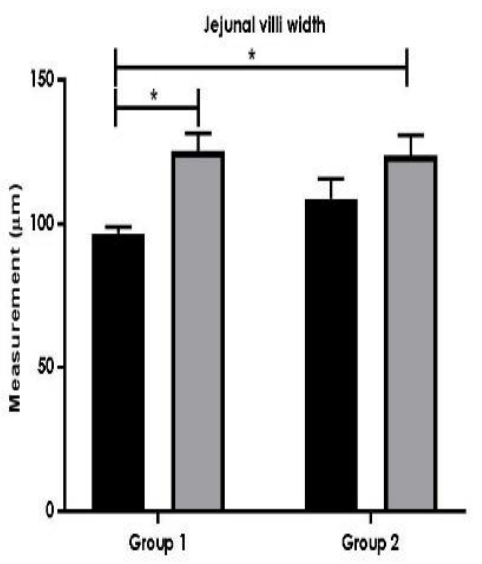

D

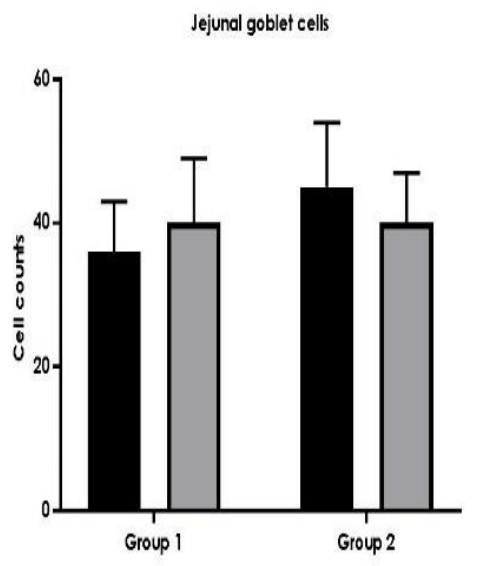

Pregnant $\square$ Non-pregnant

Figure 1. Measurement of jejunal vill height (A), villi width (B), crypt depth (C) and goblet cell counts (D) in Group 1 and 2 of pregnant and non-pregnant rats. Data presented as mean \pm IS.E.M (n=6). Results were compared between Group 1 and Group 2 of pregnant and non-pregnant rats. ${ }^{*}$ for $p<0.05$. 
There were no significant differences between the height and width of the villi, crypt depth and the number of goblet cells in the ileum of group 1 and group 2 of pregnant and non pregnant rats.

A

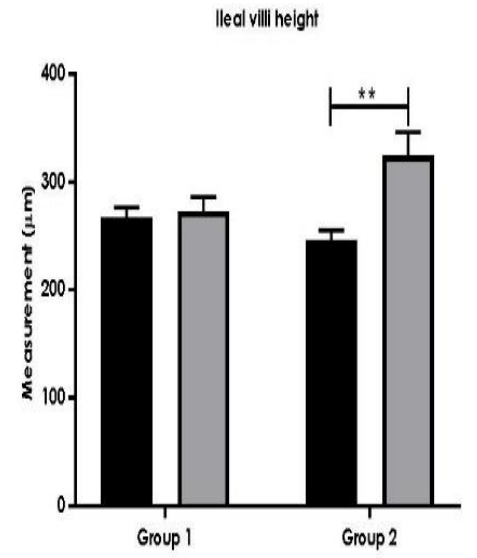

C

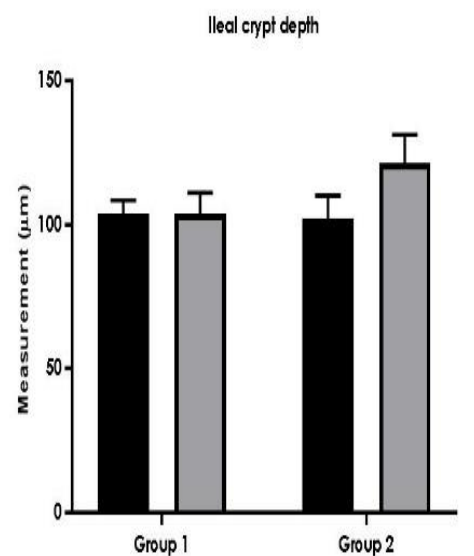

B

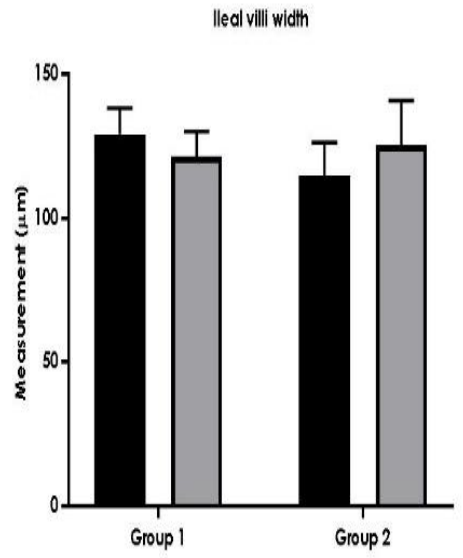

D

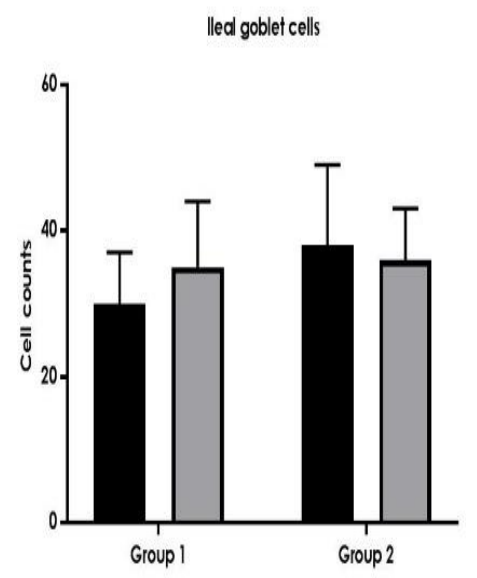

Pregnant $\square$ Non-pregnant

Figure 2. Measurement of lleal vill height (A), villi width (B), crypt depth (C) and goblet cell counts (D) in Group I and 2 of pregnant and non-pregnant rats. Data presented as mean \pm S.E.M (n=6). Results were compared between Group 1 and Group 2 of pregnant and non-pregnant rats. ${ }^{*}$ for $p<0.01$. 


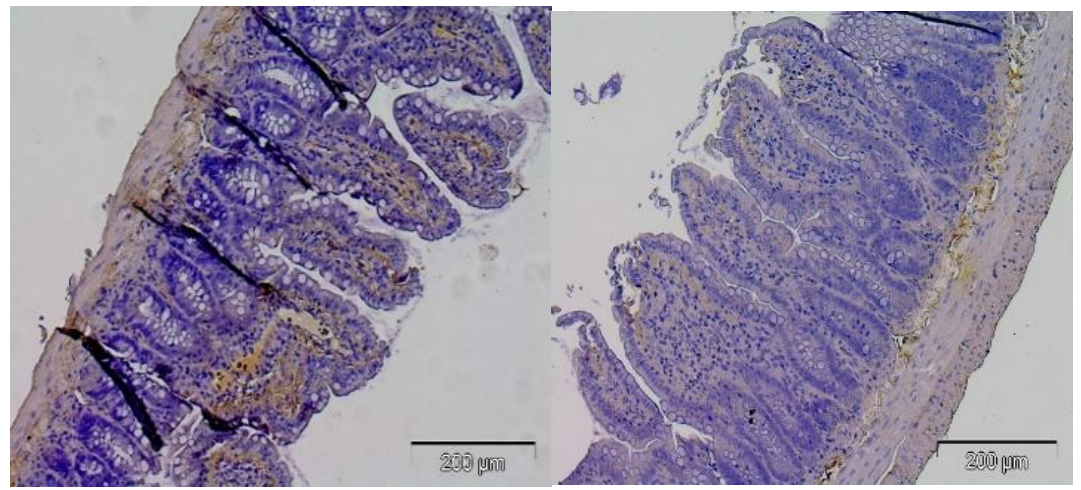

A

B

Figure 3 Displaying the expression of claudin 3 in group 1 (A) and 2 (B)

of pregnant rats

Claudin 3 expression was reduced in group 2 as compared to group 1.

Claudin 2 expression was not different between the 2 groups. (Picture not shown)

Claudin 4 was a bit faint in group 2 as compared to group 1 (Picture not shown).

Conclusion

Prolonged low dose BPA exposure throughout pregnancy have the tendency to compromise intestinal barrier in rats.

\section{ACKNOWLEDGMENTS}

Bestari Perdana grant no. 600-IRMI/DANA 5/3/BESTARI (P) (014/2018).

Universiti Teknologi MARA Committee of Animal Research and Ethics (UiTM CARE), Puncak Alam, Malaysia

Institute of Medical and Molecular Biotechnology (IMMB), Laboratory Animal Care Unit (LACU) and Centre of Pathology, Diagnostic and Research Laboratories (CPDRL) UiTM.

References

1. Lan HC, Wu KY, Lin IW, Yang ZJ, Chang AA, Hu MC. Bisphenol A disrupts steroidogenesis and induces a sex hormone imbalance through c-Jun phosphorylation in Leydig cells. Chemosphere. 2017;185:237-46 
2. Kobayashi K, Ohtani K, Kubota H, Miyagawa M. Dietary exposure to low doses of Bisphenol A: effects on reproduction and development in two generations of C57BL/6J mice. Congenital anomalies. 2010;50(3):159-70

3. Tiwari SK, Agarwal S, Chauhan LK, Mishra VN, Chaturvedi RK. Bisphenol-A impairs myelination potential during development in the hippocampus of the rat brain. Molecular neurobiology. 2015;51(3):1395-416 